\title{
Modified Variable Step Size FLOM-CMA Based on Lorentzian Function under Impulse Noise
}

\author{
Liao Xichang ${ }^{1, a}$, Lei Yingke ${ }^{2}$ \\ ${ }^{1}$ National University of Defense Technology School of electronic countermeasures Hefei, China
}

\begin{abstract}
Aiming at the problem that the traditional equalization algorithm under impulse noise is difficult to suppress impulse noise and achieve equalization, a new modified variable step size FLOM-CMA algorithm based on Lorentzian function is proposed in this paper. The proposed algorithm has modified the cost function to made full use of the phase information of signal to correct the phase rotation. Also, by adjusting the FLOM of cost function, that impulse noise is restrained effectively. Furthermore, the Lorentzian function is use to update the step size to make sure it is is appropriate for each equalization point. Simulation experiments show that, compared with FLOM-CMA and VS-FLOM-CMA, the proposed algorithm that the proposed algorithm achieves higher convergence accuracy with similar convergence speed. And under different impulse noise conditions, the robustness of the algorithm is better.
\end{abstract}

\section{Introduction}

When a digital signal is transmitted in a high-speed communication system with limited bandwidth, intersymbols-interference (ISI) will inevitably occur. At the same time, affected by noise in the transmission channel, which may cause an increase in the signal error rate (SER) and the reliability of the communication system would be affected. Therefore, the channel equalization technique is needed to compensate or reduce the distortion of signal, which is caused by objective factors[1]. The traditional equalization algorithms can be classified into non-blind and blind equalization according to whether the priori information or training sequences for transmitting signal is needed. Since the non-blind equalization algorithms require additional training sequences, and the training sequence will occupy a limited bandwidth, which is not suitable for high-speed digital communication systems[2]. In order to improve the frequency band utilization effectively, the blind equalization algorithm has been widely studied in recent years.

One of the most famous blind equalization algorithm is the Constant modulus algorithm (CMA). CMA is based on Bussgang theory and updates the equalizer weight vector by solving the optimal solution of the cost function. The algorithm is simple in structure and easy to implement, but the convergence accuracy is low and the phase signal rotation cannot be corrected[4]. And then, the modified constant modulus algorithm (MMA) is proposed to correct the phase rotation of the signal by calculating the real and imaginary parts of the signal[4], but the convergence accuracy of MMA is still low. On this basis, improved blind equalization algorithms for different signals emerge one after another[2, 4]. Most of these algorithms are proposed under Gaussian noise, however, owing to the environmental and human factors such as thunderstorm and multi-user interference, additive noise is often not subject to Gaussian distribution in wireless channels[5]. In recent years, scholars have found that noise in the actual environment often exhibits pulse characteristics and the alpha stable distribution is proved to be the best model to simulate this noise[6].

The traditional equalization algorithm performs poorly under impulse noise. In order to overcome this shortcoming, a constant modulus algorithm based on fractional lower moments (FLOM-CMA) has been proposed[7]. By adjusting the second moment in the cost function to the fractional lower moment, the FLOMCMA algorithm achieves the purpose of effectively suppressing the impulse noise and achieving blind equalization of the signal. Due to the poor convergence accuracy and speed of FLOM-CMA, by introducing the recursive least square theory, a RLS-CMA algorithm is proposed in [8] to achieve faster convergence accuracy, but it can not correct the phase rotation of signal, and the performance is affected by the constant iterative step size. Similar to MMA, a variable step size FLOM-CMA has been proposed by calculating the real and imaginary parts of the signal separately[5], which can effectively correct the phase rotation and improve the convergence speed. But algorithm has limited ability to suppress pulses. On this basis, in order to achieve better equalization effect while suppressing impulse noise, some combination algorithms based on different equalization models are constantly proposed for different conditions[9], however,

${ }^{a}$ Corresponding author: $15609698732 @ 163 . c o m$ 
the combination algorithm will bring a large amount of calculation.

In this paper, we have study the blind equalization algorithm in impulse noise and proposed a new modified variable step size FLOM-CMA. In order to suppress the impulse components, we have adjusting the fractional lower order statistics. Also, we have make full use of the phase and amplitude information of signal by calculating the real and imaginary parts respectively, so the rotation of signal have been corrected. In addition, we use the Lorentzian function to control the iteration step size of equalizer The simulation results show that the proposed algorithm not only improves the ability of pulse suppression but also achieves more precise convergence.

\section{System Model and Basic Theory}

\subsection{Equalization Model}

In wireless digital communication system, we assume that the source signal $x(n)$ are independent and identically distributed (i.i.d). After modulation, it is transmitted in the channel. In this paper, we assume the transmitted signal is the base-band signal. Then, the received signal can be defined as

$$
r(n)=\sum_{i=0}^{L-1} h_{i} x(n-i)+v(n)
$$

Where $\boldsymbol{h}=\left[h_{0}, h_{1}, \ldots h_{L}\right]$ is the unknown channel impulse response with the length of $\mathrm{L}, v(n)$ is assumed as the additive impulse noise which is introduced in the wireless channel. After the blind equalization processing, the output signal can be expressed as

$$
y(n)=\boldsymbol{w}^{\mathrm{T}} \boldsymbol{r}=\sum_{i=0}^{M-1} w_{i} r(n-i)
$$

Where $\boldsymbol{w}=\left[w_{0}, w_{1}, \ldots w_{M-1}\right]^{T}$ is an M-tap equalizer; $y(n)$ is the equalized signal, after through the decision device, we can obtain $\hat{x}(n)$, which is the decision output signal of the equalization system.

\subsection{Noise Model}

With the development of communication technology, the electromagnetic interference is often encountered in wireless channels. The Gauss noise model is no longer appropriate, and the actual noise is often considered to be subject to Alpha stable distribution. There is no closed form probability density function expression for stable distribution, so we can use the characteristic function to define it [6]

$$
\varphi(t)=\left\{\begin{array}{cc}
e^{\left\{j a t-\gamma|t|^{\alpha}\left[1+j \beta \operatorname{sgn}(t) \tan \left(\frac{\alpha \pi}{2}\right)\right]\right\}} & , \alpha \neq 1 \\
e^{\left\{j a t-\gamma|t|^{\alpha}\left[1+j \beta \operatorname{sgn}(t) \frac{2}{\pi} \lg |t|\right]\right\}} & , \alpha=1
\end{array}\right.
$$

Where $\operatorname{sgn}(\cdot)$ is the sign function, As we can see in the above equation, there is four parameters denote a
Alpha-stable distribution, which are the characteristic parameter $\alpha(0<\alpha<2)$, the scale parameter $\gamma(\gamma \geq 0)$, the symmetry parameter $\beta(-1<\beta<1)$ and the location parameter $a(a \in R)$. Separately, $\alpha$ describes the tail thickness and the size of impulse outliers; $\gamma$, similar to the variance of Gaussian noise, describes the degree of noise deviating from the mean; $\beta$ is always assume to be zero, then the Alpha-stable distribution turns to be the symmetric $\alpha$-stable $(S \alpha S)$. Research shows that $S \alpha S$ can effectively simulate the most kind of impulse noise in different transmission channel, so it is adopted in this paper.

\subsection{Fractional lower Order Moment}

Because there is no second order and higher order moments of $S \alpha S$ distribution noise, the traditional algorithm based on signal higher-order statistics can not be used in impulsive noise environment. Fractional lower order moment (FLOM), as a substitute for higher order moment, is one of the effective methods for signal processing in impulsive noise, which can be defined as[6]

$$
\begin{gathered}
\left.E \|\left. X\right|^{p}\right]=C(p, \alpha) \gamma^{p / \alpha}, 0<p<\alpha \\
C(p, \alpha)=\frac{2^{p+1} \Gamma[p+1 / 2] \Gamma(-p / 2)}{\alpha \sqrt{\pi}(-p / 2)}
\end{gathered}
$$

Where, random variable $X$ is assuming to obey the $S \alpha S$ distribution. $C(p, \alpha)$ is a constant determined FLOM $p$ and $\alpha$, and $\Gamma(x)=\int_{0}^{\infty} t^{x-1} e^{-t} d t$ is the gamma function.

If joint random variable $X_{1}, \cdots, X_{n}$ is independent and identically distributed to $S \alpha S$ distribution, we can define that $E\left[\left|X_{1}\right|^{p_{1}} \cdots\left|X_{n}\right|^{p_{n}}\right]<\infty, \sum_{i=1}^{n} p_{i} \in(0, \alpha)$.

\section{The Proposed Algorithm}

Based on the above analysis, in order to obtain better blind equalization performance, the structure of our new modified variable step-size FLOM-CMA is shown in Fig.1.

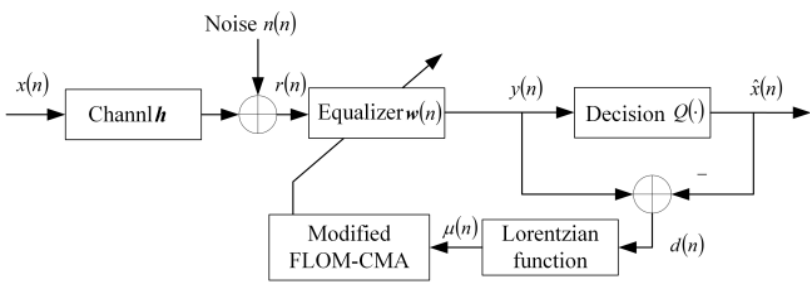

Figure 1. The structure of our proposed algorithm.

As we can see in Fig.1, after transmitted in wireless channel and affected by additive noise, the received signals $r(n)$ need to be equalized. In order to achieve the 
purpose of suppressing impulse noise, the traditional cost function of FLOM-CMA can be defined as

$$
J(\boldsymbol{w})=E\left\{\left.|| y(n)\right|^{p}-\left.R\right|^{2}\right\}
$$

Where $R=E\left\lfloor\left|x_{r}(n)\right|^{2 p} \mid / E\left\lfloor\left|x_{r}(n)\right|^{p}\right\rfloor\right.$ is he constant modulus value, $p \leq \alpha / 2$ is the FLOM. According to the steepest gradient descent method, our goal is to calculate the optimal solution of the cost function, then, iterating equalizer weight vector by the optimal solution. The FLOM is only used in the $|y(n)|^{p}$, but actually, after subtracted from the constant $R,|y(n)|^{p}$ can still be regarded as $S \alpha S$ distribution, if we use the second order moments here, the impulse components are still larger. Therefore, through comparative analysis, in order to effectively correct phase rotation and suppress impulse noise better, we have modified the cost function to be

$$
\begin{aligned}
J_{F}^{\prime}(\boldsymbol{w}) & =J_{r}(\boldsymbol{w})+J_{i}(\boldsymbol{w}) \\
& =E\left\{\left|y_{r}(n)\right|-\left.R_{r}\right|^{q}\right\}+E\left\{\left|y_{i}(n)\right|-\left.R_{i}\right|^{q}\right\}
\end{aligned}
$$

Where $y_{r}(n)$ and $y_{i}(n)$ is the real and imaginary part of $y(n) ; q \leq \alpha / 2$ is the FLOM. And for complex signals $x(n)=x_{r}(n)+j x_{i}(n)$, the constant modulus value of real and imaginary part can be defined as $\quad R_{r}=\frac{E\left\lfloor\left. x_{r}(n)\right|^{2}\right\rfloor}{E\left\lfloor\left|x_{r}(n)\right|\right]}$ and $R_{i}=\frac{E\left\lfloor\left. x_{i}(n)\right|^{2}\right\rfloor}{E\left[x_{i}(n) \mid\right]}$. Therefore, the iterative expression of equalization weight vector can be expressed as

$$
\begin{aligned}
\boldsymbol{w}(n+1) & =\boldsymbol{w}(n)-\mu \frac{\partial J^{\prime}(\boldsymbol{w})}{\partial \boldsymbol{w}} \\
& =\boldsymbol{w}(n)-\mu q c(n) \boldsymbol{r}^{*}(n)
\end{aligned}
$$

Where $\mu$ is the iterative step size of the proposed algorithm; according to the steepest gradient descent method, we can obtain the iterative error is

$$
\begin{aligned}
c(n) & =\left.|| y_{r}(n)\right|^{p}-\left.R_{r}\right|^{q-1} \operatorname{sgn}\left[e_{r}(n)\right] \operatorname{sgn}\left[y_{r}(n)\right] \\
& +\left.j|| y_{i}(n)\right|^{p}-\left.R_{i}\right|^{q-1} \operatorname{sgn}\left[e_{i}(n)\right] \operatorname{sgn}\left[y_{i}(n)\right]
\end{aligned}
$$

The advantage of the proposed algorithm is that it avoids the calculation of the second-order moments in the cost function and calculates the real part and the imaginary part of the signal separately, which can not only suppress the impulse noise better, but also make full use of the amplitude and phase information of the signal.

For blind equalization algorithms with fixed iteration step size, the sharpest problem in equalization algorithms is that there is often an inverse relationship between the convergence accuracy and the convergence speed. For example, when the iterative step size of the algorithm is large, the convergence speed would be faster, but the convergence accuracy would be reduced. But when we reduce the step size to improve the accuracy, the convergence time will be long. To solve this problem, the variable step size is very necessary. Most equalizers and filters use Sigmoid function or its deformation to iterate the step size. The variable step size algorithm based on Sigmoid function can improve the performance of blind equalization to a certain extent. However, when the decision error $d(n)=\hat{x}(n)-y(n)$ is close to 0 , the step size changes greatly, which means that the step size is still larger when it is in steady state. So in this paper, we use the Lorentzian function to control the step size of our equalization algorithm[10], the expression of our proposed variable step size is

$$
\mu(n)=\eta \lg \left\{1+\frac{1}{2}\left[\frac{d(n)}{\rho}\right]^{q}\right\}
$$

Where $\eta$ controls the range of changes; $\rho$ defines the shape when the function tends to be stable, in this paper, we set $\rho=1$. Then the equalization weight vector of the new modified variable step size FLOM-CMA algorithm in this paper can be rewrite as

$$
\boldsymbol{w}(n+1)=\boldsymbol{w}(n)-q \mu(n) c(n) \boldsymbol{r}^{*}(n)
$$

Where $\mu(n)$ and $c(n)$ are given by Eq.(12) and Eq.(11).

\section{Simulation Results}

In order to verify the performance of our proposed algorithm, we have compared the performance of FLOMCMA, VS-FLOS-MCMA[5] and the proposed algorithm in this paper under different impulse noise conditions through simulation experiments in this section. ISI is applied here to evaluate the convergence performance of each algorithm

$$
\text { ISI }=10 \lg \frac{\sum_{n=1}^{N-M+1}\left|C_{n}\right|^{2}-\left(\left|C_{n}\right|^{2}\right)_{\max }}{\left(\left|C_{n}\right|^{2}\right)_{\max }}
$$

Where $C_{n}=\boldsymbol{h}(n) \otimes \boldsymbol{w}(n)$ is the convolution of the channel impulse response and the weight vector of blind equalizer. For the $S \alpha S$ noise with scale parameter $\gamma$, the generalized signal-to-noise ration (GSNR) can be defined as $\operatorname{GSNR}=10 \lg \left(\sigma_{s}^{2} / \gamma\right)$, and $\sigma_{s}^{2}$ is the variance of the input signal $x(n)$.

The experiment mainly adopts 16QAM signal with data length $N=10000$; all three blind equalization algorithms adopt initial equalizer with length $M=9$ and center tap coefficient 1; the iteration step size for FLOMCMA is $\mu=0.0015$ and for VS-FLOS-MCMA and our proposed algorithm $\eta=0.0015$; and the fractional lower moment is $p=q=0.8$.

Firstly, we set $\alpha=1.8, \mathrm{GSNR}=25 \mathrm{~dB}$ and assume that the impulse response of channel is 


$$
\begin{aligned}
\boldsymbol{h}=[ & -0.005-0.004 j, 0.009-0.3 j, \\
& -0.0024-0.104 j, 0.864+0.52 j, \\
& -0.218+0.273 j, 0.049-0.074 j, 0.016+0.2 j]
\end{aligned}
$$

Fig. 2 is the output constellation diagram of the three algorithms after convergence. As we can see, when the algorithms converge, all the three algorithms can separate the constellation of 16QAM, but it is obvious that FLOM-CMA can not correct the phase rotation of the signal. And compared with VS-FLOM-MCMA, the degree of convergence of the proposed algorithm in this paper is ever higher.

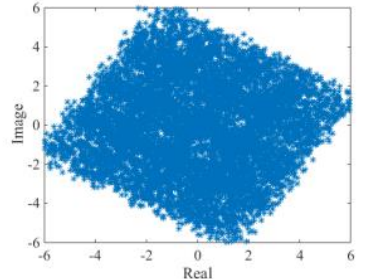

(a)Received Signal

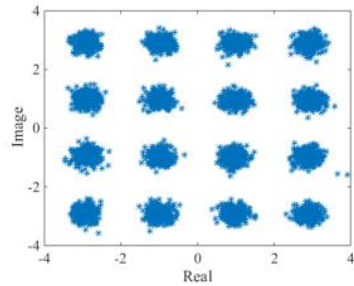

(c)VS-FLOM-MCMA

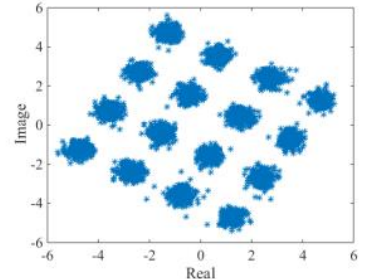

(b) FLOM-CMA

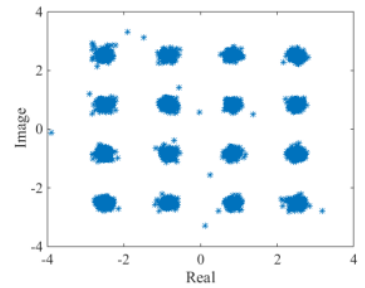

(d) The proposed algorithm
Figure 2. The constellations of different algorithm after convergence.

Secondly, we compare the convergence performance of the each algorithm in different $S \alpha S$ noise through 100 Monte Carlo experiments, and the experimental parameters remain unchanged here. We fixed $\alpha=1.8$ to compare the curves of $\mathrm{GSNR}=25 \mathrm{~dB}$ and GSNR $=20 \mathrm{~dB}$. The results is shown in Fig. 3 and Fig. 4.

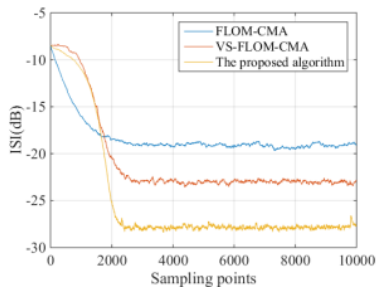

(a) GSNR=25dB

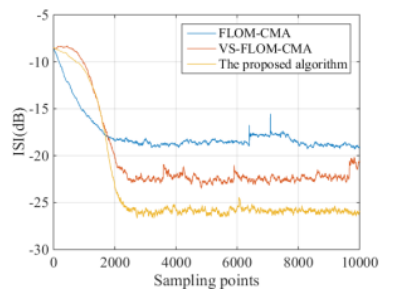

(b) GSNR=20dB.
Figure 3. The ISI curves of different algorithm

In Fig.3, we can find that both of these algorithms converge at around 2500 sampling points, which means that the convergence speed of them are similar. When the algorithms have converged, the ISI of the FLOM-CMA is nearly $-19 \mathrm{~dB}$; by use the variable-step size algorithm, the VS-FLOM-MCMA has improved the ISI curves about $4 \mathrm{~dB}$ with the condition that the speed of convergence does not increase significantly. But for our proposed algorithm, the step size corresponding to the equalization of each sampling point can be calculated more accurately by the variable step size algorithm based on Lorentzian function. So, as its shown in Fig.3, the ISI of our algorithm is about $-28 \mathrm{~dB}$, which is better than the other two algorithms. Comparing Fig.3(a) and Fig.3(b), when the GSNR becomes smaller, the performance of the three algorithms have dropped a little, but our proposed algorithm is still better than FLOM-CMA and VSFLOM-CMA.

\section{Conclusion}

In order to achieve better performance blind equalization while effectively suppressing impulse noise, we have mainly studied the blind equalization algorithm in $S \alpha S$ noise and proposes a new modified variable step size FLOM-CMA algorithm, which is based on Lorentzian function. The proposed algorithm adjusts the FLOM of the cost function, and calculates the real and imaginary parts of the signal separately. Therefore, the phase rotation of the signal is effectively corrected, and at the same time, the step size is updated according to the Lorentzian function, a higher convergence precision is achieved under the condition that the convergence speed does not increase. The performance of the proposed algorithm is verified by several experiments, and the results show that the algorithm can not only suppress the pulse noise better, but also achieve higher convergence accuracy.

\section{References}

1. Y. Sun, F. Wang, C. Jia, \& Z Liu, Blind equalization algorithm for different eigenvalue spread channels. Wireless Personal Communications. 101, 2, 1-16(2018).

2. M. T. M. Silva, Arenas-Garcia J, A soft-switching blind equalization scheme via convex combination of adaptive filters. IEEE Transactions on Signal Processing. 61, 5, 1171-1182(2013)

3. A. J. V. D. Veen, A. Paulraj, An analytical constant modulus algorithm. Signal Processing IEEE Transactions on. 44, 5, 1136-1155(1996).

4. A. W. Azim, S. Abrar, A.Zerguine, A. K. Nandi, Performance analysis of a family of adaptive blind equalization algorithms for square-qam. Digital Signal Processing. 48, C, 163-177(2016).

5. J. Ma, T. Qiu, R. Li, J. Li, Variable step-size modified blind equalization algorithm based on fractional lower order statistics under impulsive noise. IEEE International Conference on Computer and Communications. pp:1367-1371(2017).

6. C. L. Nikias, Signal processing with alpha-stable distributions and applications. John Wiley \& Sons, Inc(1995).

7. M. Rupi, P. Tsakalides, E. D. Re, C. L. Nikias, Constant modulus blind equalization based on fractional lower-order statistics. Signal Processing. 84, 5, 881-894(2004).

8. Y. Guo, T. S. Qiu, H. Tang, Y. W. Park, Constant modulus algorithm for blind equalization under 
impulsive noise environments. Journal on Communications. 30, 4, 35-40(2009).

9. J. Ma, T. Qiu, L. I. Rong, N. Xia, L. I. Jingchun, Concurrent blind equalization algorithm based on probability density function matching and fractional lower order moments. Journal of Electronics \& Information Technology. 39, 7,1532-1538(2017)

10. L. Y. Zeng, X. U. Hua, T. R. Wang, Low computational complexity variable step-size clms algorithm based on lorentzian function. Systems Engineering \& Electronics. 38, 5, 998-1003(2016) 